\title{
A new pop-up experience opens in London
}

Align Technology, Inc. has launched a new Invisalign Connect Pop-Up in Westfield Shopping Centre Stratford. The immersive Invisalign Pop-Up experience has been created to re-invent the way consumers interact with the Invisalign brand, access information about teeth straightening and connect with Invisalign doctors in the local area network.

By bringing the Invisalign brand closer to consumers - and showcasing Invisalign technology in an engaging, non-clinical environment - visitors to the Invisalign Connect Pop-Up can explore the potential of clear aligner therapy, learn what to expect during treatment and how to get started with an Invisalign trained doctor. The Pop-Up team will also be on hand to guide visitors and answer their questions about the Invisalign system.

The previous Invisalign Pop-Up opened in July and was the first time Invisalign had truly engaged consumers in a physical environment as a brand. This has helped the company to refine the pop-up experience and better inspire and engage consumers.

Westfield Pop-Up visitors can even try on a new smile in approximately 60 seconds.

Thanks to the latest SmileView technology,

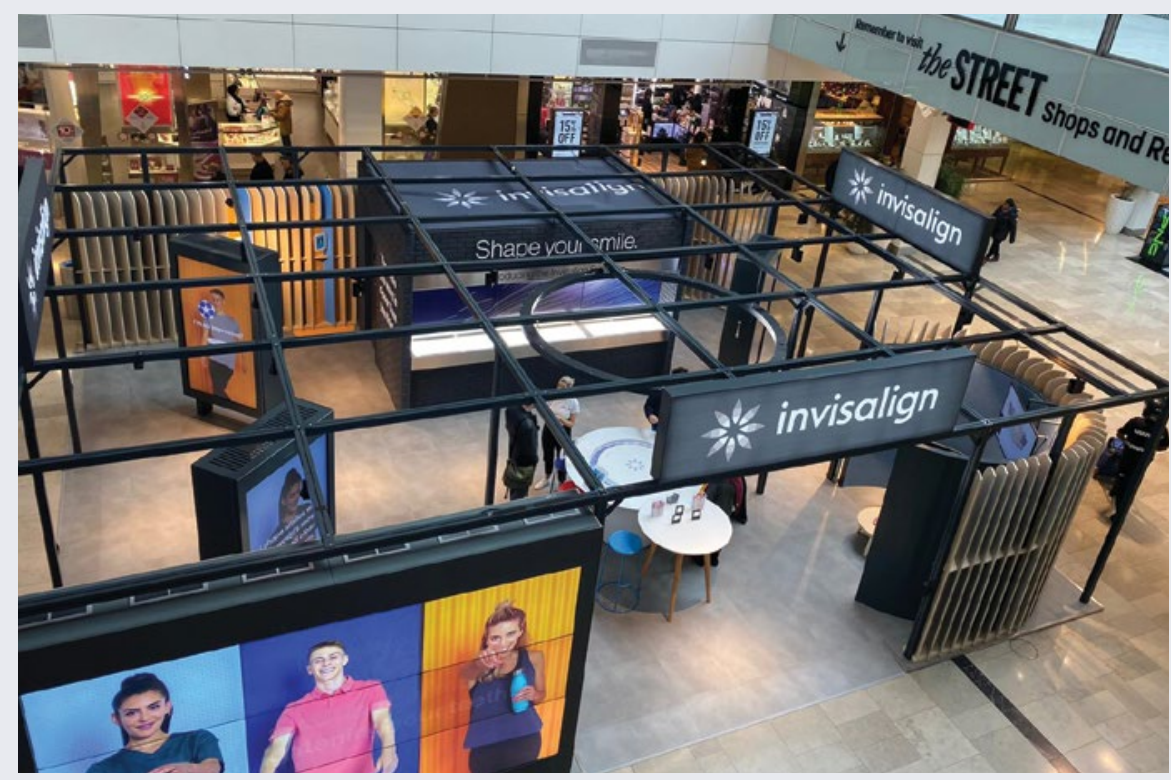

developed by Align, consumers can take a selfie and see a non-clinical visualisation of what their new smile could look like in their own photo. If they like what they see, they can be connected to an Invisalign doctor of their choice for a free initial consultation to determine if they are a good candidate for Invisalign treatment.

The Invisalign Connect Pop-Up also brings to life real patient stories from the Invisalign Smile Squad - Invisalign social media influencers and brand ambassadors - who have embarked on the Invisalign treatment journey and who are excited to share their stories.

The Invisalign Connect Pop-Up is located in the North Gallery at Westfield Stratford Shopping Centre, Olympic Park, Montfichet Road, Stratford, London, E20 1EJ. It is open on Monday-Friday 10 am-10 pm and weekends $12-6 \mathrm{pm}$.

\section{Put your patients in pole position}

Patient positioning plays a key role in the acquisition of high-quality diagnostic images. So why not ensure you can get the optimal outcome every time by choosing the CS 9600 CBCT system from Carestream Dental?

With its Live Positioning Assistant, the system helps ensure that patients are always in the best position possible for greater accuracy and exam precision. This helps reduce the amount of retakes needed to get the perfect shot.

Furthermore, the system records patient parameters automatically; so follow-up imagery is repeatable with a single touch.

For more information, contact Carestream Dental on 08001699692 or visit www.carestreamdental.co.uk.

\section{Ensuring dental implant success}

Minimising the risk of infection helps ensure the success of dental implant therapy, particularly for patients with systemic health issues. TBR offers a cutting-edge implant system designed to support both hard and soft tissue integration.

The Z1 features an innovative zirconia collar that acts as an antibacterial shield to protect the crestal bone and the gingiva from iatrogenic inflammation and infection. This encourages the soft tissue to heal around the implant in a way that mimics natural gingival growth for a truly functional and aesthetic result.

With a $98.6 \%$ success rate, why wouldn't you choose the Z1 for your implant patients? Call today for further details.

For more information on the Z1 implant, visit tbr.dental, email support@denkauk. com or call 08007076212 